\title{
THE COMPRESSION OF BONDED RUBBER DISKS
}

\author{
M. L. Anderson, P. H. MotT, ${ }^{*}$ C. M. Roland \\ Chemistry Division, Naval Research Laboratory, Washington, DC 20375
}

\begin{abstract}
Compressive stress-strain measurements are reported for bonded disks of natural rubber having shape factors $(=d / 4 t$, where $d$ is the diameter and $t$ the thickness) ranging from 0.87 to 12.95 . The data are compared to various models for compressed rubber, utilizing the tensile and bulk moduli determined for this material. We find that for thin disks, the measured stiffness is less that the theoretical predictions by roughly a factor of two. A similar discrepancy was found previously, both for elastomers and semi-crystalline polymer films. However, for smaller aspect ratios, the difference between experiment and theory is less than the measurement error.
\end{abstract}

\section{INTRODUCTION}

There are many practical situations in which a soft, nearly incompressible material, such as rubber, is bonded and compressed between rigid plates. Applications include mechanical ${ }^{1-4}$ and electrostriction measurements, ${ }^{5-7}$ civil engineering structures such as base isolation bearings in bridges and buildings, ${ }^{8,9}$ and medical uses such as periodontal membranes. ${ }^{10}$ The stress-strain relationship for bonded rubber in compression is complex, and notwithstanding its wide use, the analysis remains controversial.

The simplest and most popular analysis is due to Gent and Lindley. ${ }^{11}$ The deformation was assumed to have a parabolic profile, with the total stress the superposition of two independent forces: (i) normal stresses due to uniform compression, corresponding to the 'slip' condition of rubber between lubricated plates, and (ii) a shear stress arising from the constraints due to bonded ends. If the material is incompressible and the deformation is small enough to remain in the linear range, Gent and Lindley ${ }^{11}$ derived the 'apparent' Young's modulus as

$$
E_{A}=E\left(1+2 S^{2}\right)
$$

for cylindrical disks, where $E$ is Young's modulus of the material and $S$ is the shape factor, defined as the ratio of one loaded area to the force-free area $(=d / 4 t$, where $d$ is the diameter and $t$ the thickness). For very thin sheets (large $S$ ), bulk volume changes become important, contradicting the assumption of incompressibility. To account for this, Gent and Lindley ${ }^{11}$ reasoned empirically that the apparent modulus can be represented by

$$
\frac{1}{E_{A}}=\frac{1}{E\left(1+2 S^{2}\right)}+\frac{1}{B}
$$

where $B$ is the bulk modulus.

Using the same basic assumptions of superposition of two forces and a parabolic profile, Gent $^{12,13}$ derived an expression using linear elasticity which incorporated near incompressibility. The apparent Young's modulus was found to be

$$
E_{A}=E+B \frac{I_{2}(\xi S)}{I_{0}(\xi S)}
$$

\footnotetext{
* Corresponding author. Ph: 202-767-1720; Fax: 202-767-0594; email: phm@xbt.nrl.navy.mil
} 
where $I_{2}$ and $I_{0}$ are the modified, or hyperbolic, Bessel functions of the first kind, of order two and zero, respectively. The parameter $\xi$ is defined as

$$
\zeta=\left[\frac{24 E}{(1+v) B}\right]^{1 / 2}
$$

where $v$ is Poisson's ratio. Horton et al. ${ }^{14}$ eliminated the assumption of a parabolic profile, and obtained a different expression, again using the superposition of normal and shear forces:

$$
\frac{1}{E_{A}}=\frac{1}{E}\left(1-S \sqrt{2 / 3} \tanh \frac{\sqrt{3}}{S \sqrt{2}}\right)+\frac{1}{B}
$$

For sufficiently large, thin sheets, $S \rightarrow \infty$, and an expression can be derived from linear elasticity that is independent of the sample dimensions,${ }^{6}$ by assuming that strains are confined to the applied load direction:

$$
E_{A}=\frac{3(1-v)}{(1+v)} B
$$

This relation serves as an upper bound to $E_{\mathrm{A}}$, and approaches the bulk modulus as $v \rightarrow 1 / 2$.

These various models are compared in Figure 1, for an elastomer with $B=2000 E$. At intermediate shape factors, $2<S<9$, the models predict essentially identical behavior. For tall cylinders, $S<2$, the three Gent models (Equations 1, 2 and 3) converge; but the Horton et al. model (Equation 5) predicts a somewhat higher apparent modulus. For flat cylinders, $S>9$, the incompressible model (Equation 1) increases without bound with increasing shape factor; the other models (Equations 2, 3 and 5) all approach the bulk modulus, somewhat more slowly for Equation 3 than the others. For a very thin disk $(S=300)$, the apparent moduli from Equations 3 and 5 are $93 \%$ and $99 \%$ of the bulk modulus, respectively. To some degree, this justifies the empirical reasoning behind Equation 2. Interestingly, Equations 2 and 5 are equivalent at high values of the shape factor: to account for bulk volume changes, Horton et al. have applied the same empirical reasoning as Gent and Lindley.

Experimental assessments of these theoretical predictions have been scarce, and most papers introducing these models do not compare their predictions to experimental results. Gent and Lindley ${ }^{11}$ measured the apparent compressive modulus for a gum natural rubber, and found that it was in reasonable agreement with Equation 2 for $S<9$, although at larger values of $S$, the measured $E_{\mathrm{A}}$ were approximately half that from Equation 2. The discrepancy was attributed to uncertainty in the bulk modulus. These results are consistent with recent electrostriction measurements, ${ }^{15}$ wherein a large electric field $($ ca. $10 \mathrm{MV} / \mathrm{m})$ is applied across a thin film $(S \approx 230)$ of semi-crystalline poly(vinylidene fluoride) terpolymers. It was found that the apparent modulus is overestimated by a factor of two by the compressible theories (Equations 2, 3, and 6). 


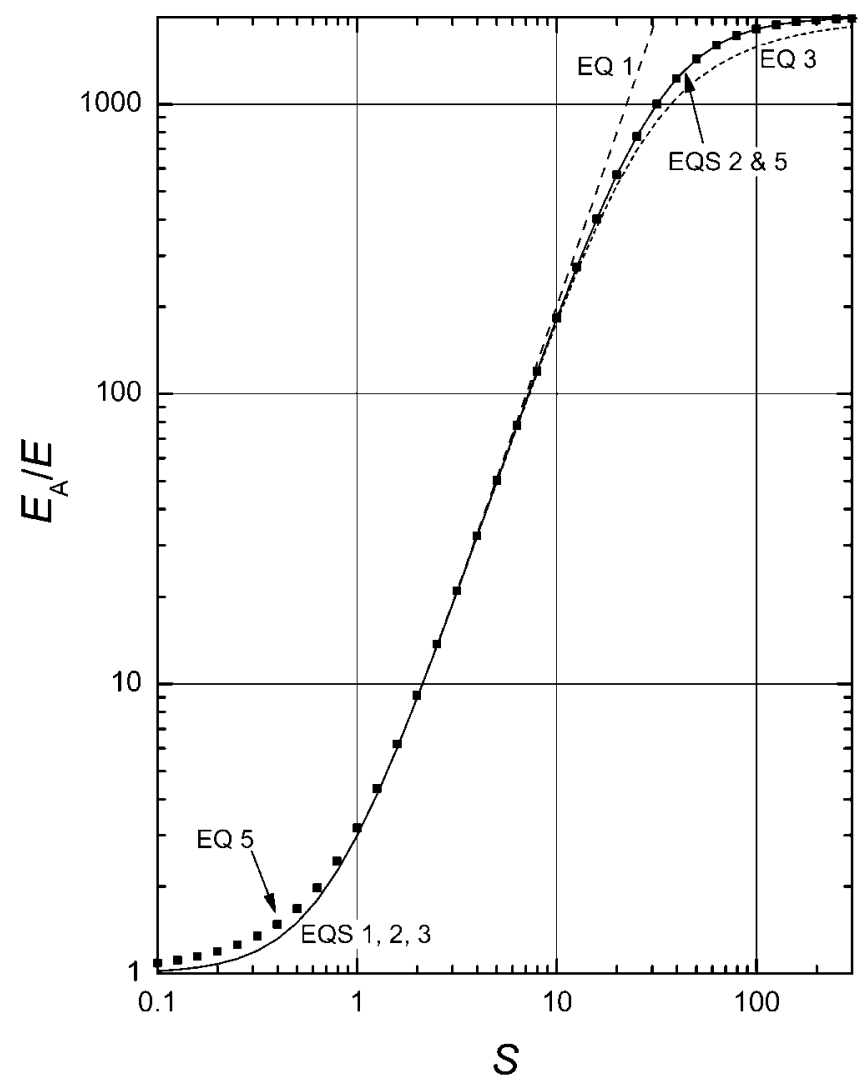

FIG. 1. - Comparison of the apparent modulus $E_{\mathrm{A}}$ from the various models: incompressible ${ }^{11}$ (Equation 1); empirically compressible ${ }^{11}$ (Equation 2); Gent's analytical compressible ${ }^{12,13}$ (Equation 3); and Horton's analytical compressible ${ }^{14}$ (Equation 5).

Mott and Roland ${ }^{16}$ compressed bonded natural rubber and polybutadiene cylinders, and determined that the deformation profile was not parabolic. Nevertheless, they found that for shape factors near unity, the apparent modulus was in good agreement with Equation 1. Fukahori et al. ${ }^{17}$ measured the creep of rubber bearings of varying shape factor, and concluded that diffusion of migratory compound ingredients (e.g., plasticizers) governed the mechanical response.

Other efforts to verify the various theories have been by finite element (FE) calculations. ${ }^{18,19}$ Morman and $\operatorname{Pan}^{20}$ compared FE results for $S=0.56$ over a wide range of strains, and found them to be in accord with Equation 1 when the compressive strain was less than $5 \%$. Hasshim et al. ${ }^{21}$ employed a simplifying assumption valid only for filled rubber, ${ }^{22}$ to model the compression of filled rubber as a homogeneous deformation. Their approximation was found to work well for $0.5 \leq S \leq 8$. Yeoh and coworkers ${ }^{13}$ carried out FE calculations of a 'typical' rubber with Poisson's ratio of 0.4995 (i.e., $B=333 E$ ), finding good agreement with Equation 3. Imbimbo and Luca ${ }^{23}$ reported agreement of the stress distribution predicted by Equation 1 only for high shape factors, $S>20$. They pointed out that the stress discontinuity at the cylinder edge, which is not captured by any of the above models, requires special effort to be accounted for in a finite element mesh. For larger strains, another problem is the difficulty of fitting the strain energy function, limiting the ability of FE calculations to describe the non-linear mechanical properties of rubber. ${ }^{18}$

We present herein new experimental results for the apparent compressive modulus of bonded gum rubber disks, measured at low strains in equilibrium. The actual Young's modulus $E$ was 
measured in tension, using strips cut from the same sheets. The bulk modulus, and thereby Poisson's ratio, were determined from pressure-volume measurements. These results, along with some previously published experimental data, ${ }^{11}$ are compared to the models.

\section{EXPERIMENTAL}

Deproteinized natural rubber (H. A. Astlett Co.) was mixed with $2.0 \mathrm{phr}$ dicumyl peroxide using a two-roll mill. Curing was carried out by compression molding at $160 \mathrm{C}$ for either $30 \mathrm{~min}$ utes (3.6 and $1.6 \mathrm{~mm}$ thick sheets) or 60 minutes ( $8.4 \mathrm{~mm}$ thick sheets). Disks of varying diameter and strips $(12 \times 152 \mathrm{~mm})$ were cut from the cured sheets.

The rubber disks were bonded to $19 \mathrm{~mm}$ thick steel plates using cyanoacrylate adhesive. Compressive load-displacement curves were measured with an Instron 4206, fitted with a linear voltage differential transducer (LVDT). The LVDT was mounted directly on the steel plates, eliminating the need to correct for the compliance of the load frame. The data were obtained stepwise in retraction, with a maximum load of $50 \mathrm{kN}$ for the largest diameter sample. The relaxation time was less than one minute. To verify that the material was in mechanical equilibrium, some reversing steps were made with increasing load. Conventional tensile stress-strain measurements were also performed, with the displacement measured using a Wallace optical extensometer. The strain rate was $0.0167 \mathrm{~s}^{-1}\left(1.0 \mathrm{~min}^{-1}\right)$, for which the extension and retraction curves superimposed. Decreasing the strain rate by a factor of ten did not alter the measurement, corroborating the attainment of equilibrium.

Dilatometric measurements employed a Gnomix apparatus. ${ }^{24}$ The method is based on the confining fluid technique, with the sample surrounded by mercury. The maximum hydrostatic pressure of the test is $200 \mathrm{MPa}$.

\section{RESULTS AND DISCUSSION}

\section{BULK MODULUS}

Figure 2 shows the pressure $P$ as a function of volume $V$ for nearly adiabatic compression, from 10 to $200 \mathrm{MPa}$. The temperature $T$ range is 20.6 to $53.8 \mathrm{C}$. The data were fit to the Tait equation, ${ }^{25}$

$$
V=\left(V_{0}+V_{1} T+V_{2} T^{2}\right)\left[1-0.0894 \ln \left(1+\frac{P}{b_{0} \exp \left(-b_{1} T\right)}\right)\right]
$$

TABLE I

Tait Equation (Equation 7) Fit Parameters From Figure 2

\begin{tabular}{cc} 
Parameter & Value \\
\hline$V_{0}$ & $872.53 \mathrm{~mm}^{3}$ \\
$V_{1}$ & $0.61349 \mathrm{~mm}^{3} / \mathrm{C}$ \\
$V_{2}$ & $-0.0001 \mathrm{~mm}^{6} / \mathrm{C}^{2}$ \\
$b_{0}$ & $185.24 \mathrm{MPa}^{-1}$ \\
$b_{1}$ & $0.00443 \mathrm{C}^{-1}$ \\
\hline
\end{tabular}

where $V_{0}, V_{1}, V_{2}, b_{0}$, and $b_{1}$ are material constants. The fits are included in Figure 2, with the Tait parameters listed in Table I. The bulk modulus, calculated as $B=-V \partial P / \partial V$ from the fit to Equation 7, along with the measured values calculated as $B=-V \Delta P / \Delta V$ from the data, is shown for ambient temperature (24.3 C) in Figure 3. At zero pressure, $B=1870 \mathrm{MPa}$. 


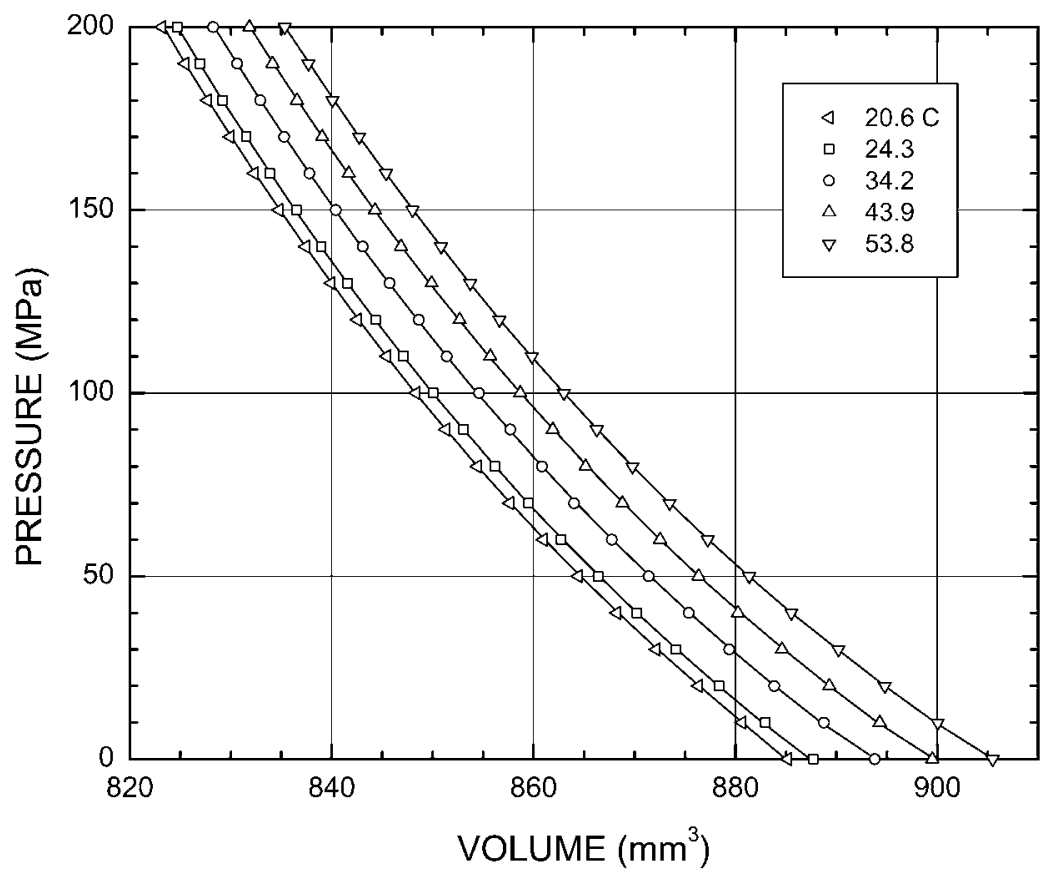

FIG. 2. - Compressibility of deproteinized natural rubber, crosslinked with $2.0 \mathrm{phr}$ dicumyl peroxide.

The indicated temperatures are averaged over each compression, with a variation of $\pm 0.5 \mathrm{C}$.

The lines represent the Tait equation ${ }^{25}$ (Equation 7). The sample mass was $0.8521 \mathrm{~g}$.

The bulk modulus for peroxide cured natural rubber was previously measured by Wood and Martin $^{26}$ and Moonan and Tschoegl. ${ }^{27}$ At ambient pressure, they obtained $B=1946 \mathrm{MPa}$ and $1690 \mathrm{MPa}$ respectively, which bracket the present measurement. We do not expect significant variation of $B$ with crosslink density, so the differences probably reflect experimental uncertainties.

\section{TENSION}

A representative tensile (true) stress versus strain plot is displayed in Figure 4. Young's modulus (determined from the initial slope) was approximately $1.5 \%$ lower for the retraction data than for extension. Although this is within the experimental error, the retraction results were used, since they better reflect mechanical equilibrium. ${ }^{28,29}$ The variation among samples was less than $10 \%$. The average Young's modulus, $1.07 \mathrm{MPa}$, was used for $E$ in all calculations.

Poisson's ratio was then calculated from

$$
v=1 / 2-\frac{E}{6 B}
$$

The result, $v=0.4999$, is somewhat closer to incompressible than the value employed by Yeoh and coworkers ${ }^{13}$ for finite element calculations of the deformation of bonded rubber sheets. 


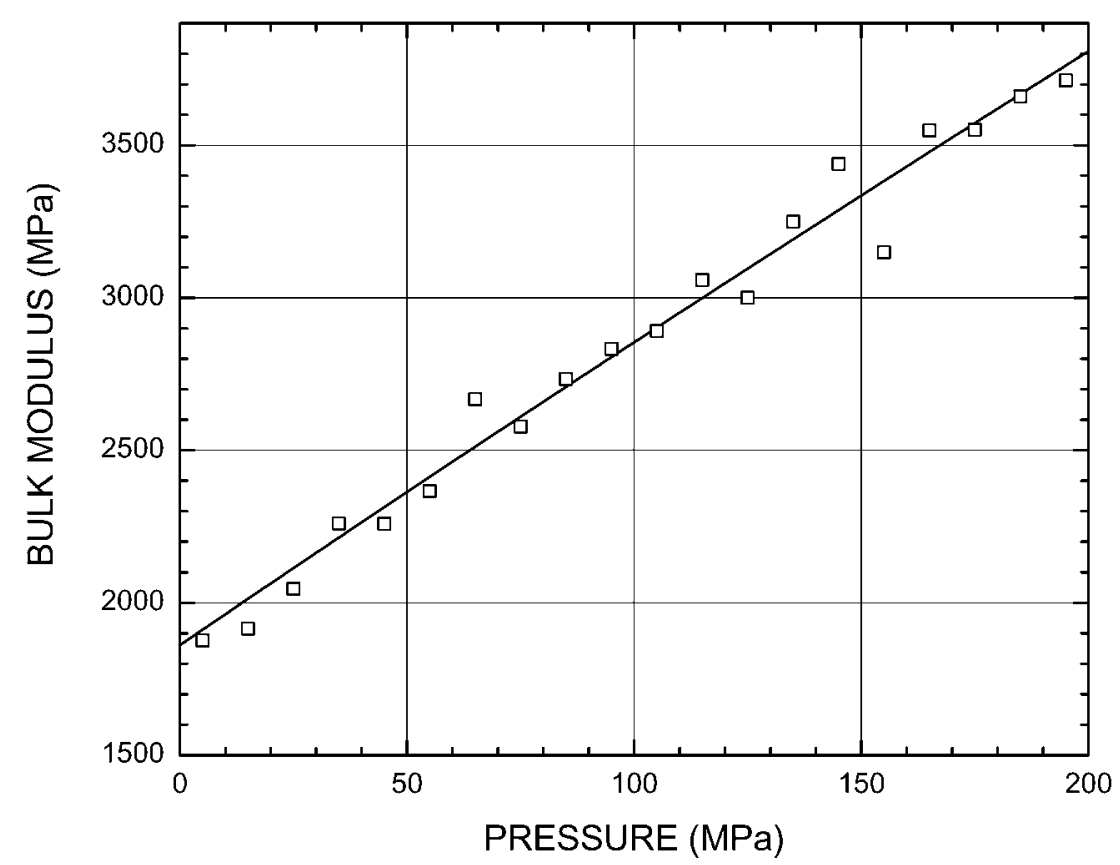

FIG. 3. - Pressure-dependent bulk modulus at 24.3 C, using $B=-V \Delta P / \Delta V$, calculated from the data in Figure 2. The line is the derivative of the fitted Tait equation.

\section{BONDED COMPRESSION}

The measured true stress is plotted in Figure 5 for the bonded disks in compression. The curves are essentially linear for compressive strains less than $5 \%$. The curves were fit to parabolas, and the apparent moduli $E_{\mathrm{A}}$ taken from the derivatives at zero strain. Figure 6 compares this experimental data to the theories. For clarity, the empirical compressible theory (Equation 2) has been omitted, since it predicts the same behavior as the incompressible theory (Equation 1) at lower values of $S$, and as the Horton et al. theory (Equation 5) at higher $S$. At low values of $S$, the data agrees with the theories within experimental error. For $S>3$, the data consistently fall below all the calculated curves. In this range, the theories overestimate the modulus by roughly a factor of two.

For comparison, data taken from Gent and Lindley ${ }^{11}$ are displayed in Figure 7. Again, the lines show the incompressible model (Equation 1), Gent's analytical model (Equation 3), and the Horton et al. model (Equation 5). For the latter two curves, the bulk modulus of the sulfur-cured gum natural rubber compound was assumed to be $1960 \mathrm{MPa}$, as done originally by Gent and Lindley. This is 5\% larger than the present measurement. Again, at higher shape factors, the experimental data fall below all the theoretical curves, by approximately a factor of two. However, for low and intermediate shape factors, $S<9$, Equation 5 describes the data quite well. 


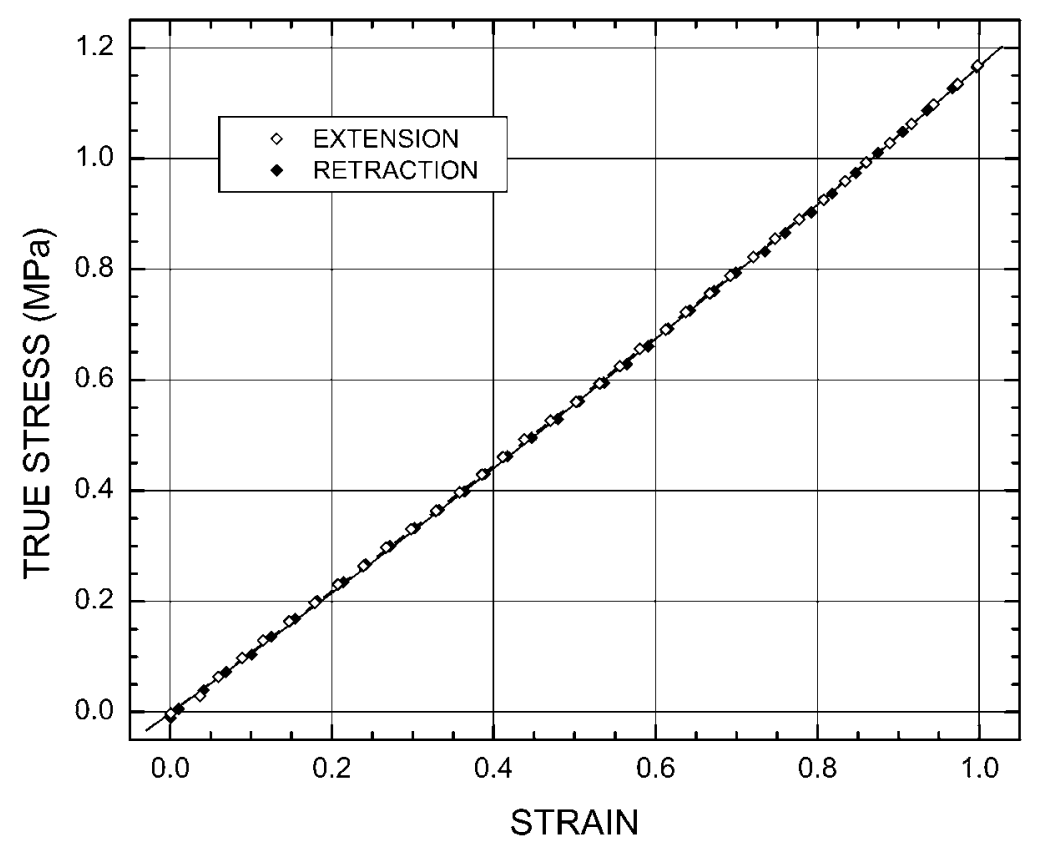

FIG. 4. — True stress as a function of strain, measured in tension. The line represents a second-order polynomial.

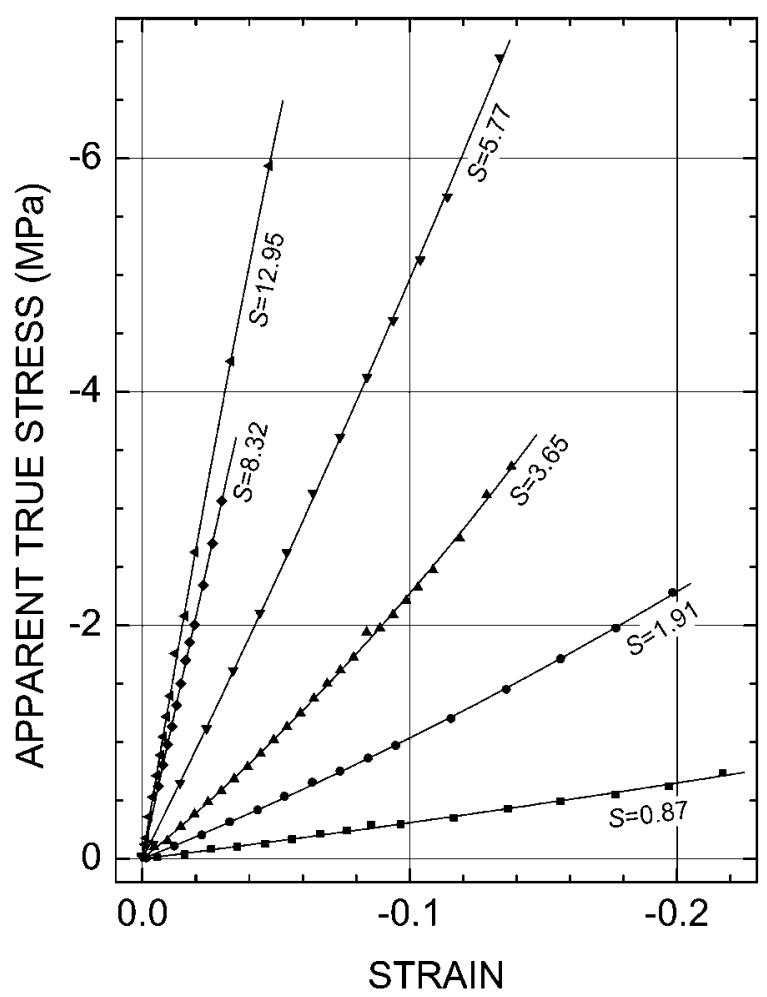

FIG. 5. - Apparent true stress as a function of compressive strain for bonded rubber having the indicated shape factors. The lines are second-order polynomial fits. 


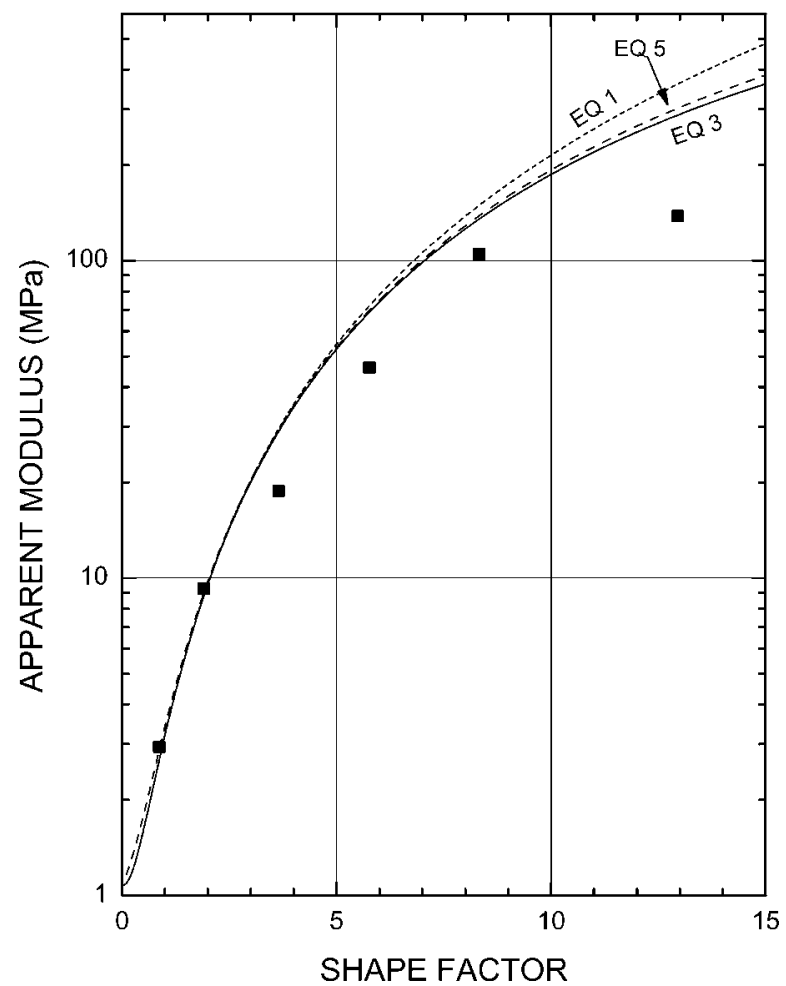

FIG. 6. - Comparison of the experimental data measured herein to the various models.

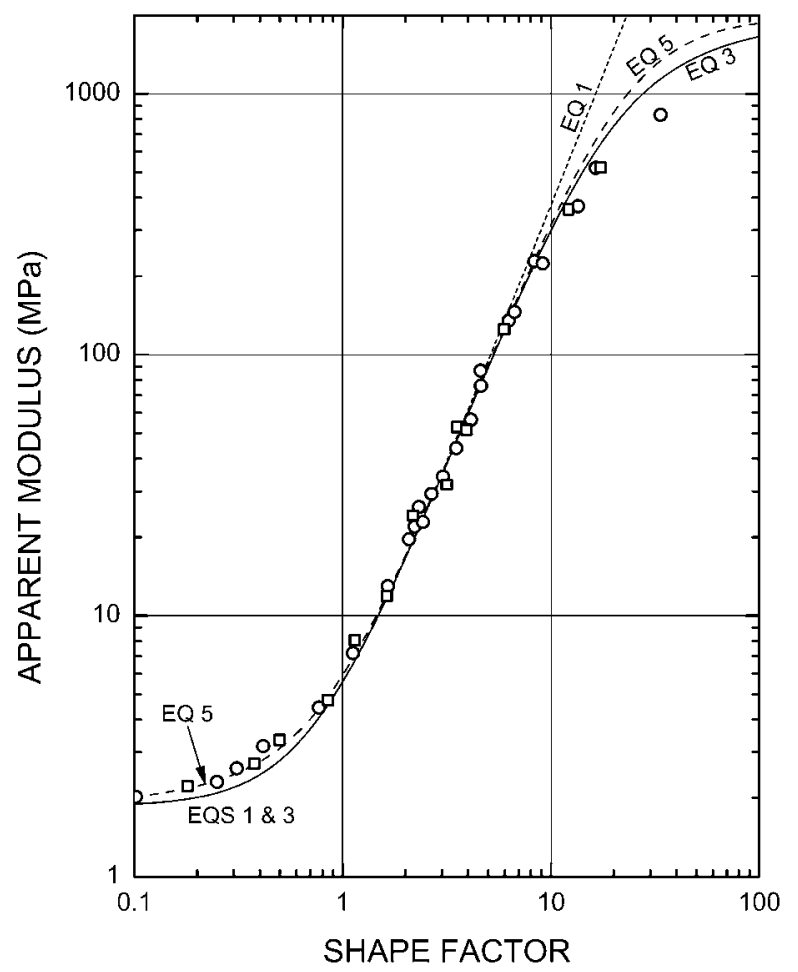

FIG. 7. - Comparison of the data from Gent and Lindley ${ }^{11}$ to the various models. 


\section{SOURCES OF ERROR}

The disagreement between the theories and experimental data at high shape factors (i.e., $S>9$ ) may be due to the high shear strains near the cylinder edge, which can give rise to additional internal stresses that are unaccounted for by the models. The Gent et al. theory ${ }^{30}$ predicts the shear strain along the bonded surface to be

$$
\gamma=6 S e \frac{r}{a}
$$

where $e$ is the compressive strain, $r$ is radial position, and $a$ the sample radius. For a shape factor of 12.95 and a 5\% compressive strain, $\gamma$ at the sample edge is predicted to be 3.89 . This is beyond the bounds for linear elastic behavior in rubber; moreover, in this range natural rubber can strain-crystallize ${ }^{31-33}$ Although this estimate cannot be correct at the edge, since a free surface cannot apply shear tractions, measurements have shown good agreement over most of the bonded surface. ${ }^{30}$ Since finite element calculations also ignore this discontinuity, ${ }^{13}$ their agreement with the models does not serve as a verification thereof.

Another potential source of error is the $0.1 \mathrm{~mm}$ variation in thickness of the rubber sheet, which introduces some uncertainty into the compressive strain. This error will increase as $S$ increases. Concerning the bonding of the rubber sheets, there was no indication of failure (e.g., cracks or delamination) in either the rubber or the adhesive bond, nor was there hysteresis in the stress-strain curve when the strain increments were reversed.

\section{CONCLUDING REMARKS}

The Horton et al. model (Equation 5) offers the best accuracy at the lowest shape factors. However, the incompressible solution (Equation 1) is simple and within $11 \%$ of the Horton et al. model for $S \leq 2$. At intermediate shape factors $(2>S>9)$, where all the models agree, the Gent and Lindley data are within the experimental uncertainty of the theoretical curves. The present measurements, however, fall below the predictions. At large shape factors $(S \geq 9)$, none of the calculations satisfactorily describe the experimental results. At intermediate shape factors, the cause of the discrepancy between the present measurements and the Gent and Lindley data is unclear. At high shape factors, however, the two experiments are in accord, and also agree with experimental results from electrostriction measurements. ${ }^{15}$ Of course, the vinylidene fluoride films used in the latter are semi-crystalline, and substantially less 'incompressible' than the present gum elastomer. Nevertheless, the failure of existing models at high shape factor indicates a need for further analytical developments.

\section{ACKNOWLEDGEMENTS}

We are indebted to Dr. R. Casalini for carrying out the dilatometric measurements, and to Professor G. S. Buckley for preparing some of the samples. This work was supported by the office of Naval Research and the Naval Sea System Command.

\section{REFERENCES}

${ }^{1}$ F. P. Wolf, Polymer 13, 347 (1973).

${ }^{2}$ P. H. Mott and C. M. Roland, Macromolecules 29, 6941 (1996).

${ }^{3}$ Erman and J. E. Mark, "Structures and Properties of Rubberlike Networks," New York: Oxford University Press, 1997. 
${ }^{4}$ A. N. Gent, "Elasticity in Engineering with Rubber: How to Design Rubber Components," 2 ${ }^{\text {nd }}$ edition. A.N. Gent, Ed., New York: Hanser Verlagr, 2001, Ch. 3.

${ }^{5}$ J. T. Garrett, C. M. Roland, A. Petchsuk, and T. C. Chung, Appl. Phys. Let. 83, 1190 (2003).

${ }^{6}$ T. Furukawa and K. Matsumoto, Jpn. J. Appl. Phys. 31, 840 (1992).

${ }^{7}$ G. S. Buckley, C. M. Roland, R. Casalini, A. Petchsuk, and T. C. Chung, Chem. Mat. 14, 2590 (2002).

8“Earthquake Resistant Construction Using Base Isolation," N. J. Raufaste, Ed., Gaithersburg, MD, NIST special publication 1992, p.832.

${ }^{9}$ C. J. Derham, A. G. Thomas, J. M. Eidinger, and J. M. Kelly, Rubber Chem. Technol. 53, 186 (1980).

${ }^{10}$ J. L. Synge, Philos. Trans. R. Soc. A231, 435 (1933).

${ }^{11}$ A. N. Gent and P. B. Lindley, Proc. Inst. Mech. Engrs. 173, 111 (1959).

${ }^{12}$ A. N. Gent, Rubber Chem. Technol. 67, 549 (1994).

${ }^{13}$ O. H. Yeoh, G. A. Pinter, and H. T. Banks, Rubber Chem. Technol. 75, 549 (2002).

${ }^{14}$ J. M. Horton, G. E. Tupholme, and M. J. C. Gover, J. Appl. Mech. 69, 836 (2002).

${ }^{15}$ C. M. Roland and J. T. Garrett, in "Nonlinear Dielectric Phenomena in Complex Liquids," S.J. Rzoska and V. Zheleny, Eds., Dordrecht, The Netherlands, Kluwer Academic. Publishers, in press.

${ }^{16}$ P. H. Mott and C. M. Roland, Rubber Chem. TeChnOL. 68, 739 (1995).

${ }^{17}$ Y. Fukahori, W. Seki, and T. Kubo, RubBER ChEM. TEChNOL. 69, 752 (1996).

${ }^{18}$ D. J. Charlton, J. Yang, and K. K. Tek, Rubber Chem. Technol. 67, 481 (1994).

${ }^{19}$ R. H. Finney, "Finite Element Analysis," Chapter 9 in Reference 4.

${ }^{20}$ K. N. Morman and T. Y. Pan, Rubber Chem. TeChnOL. 61, 503 (1988).

${ }^{21}$ S. Hasshim, J. J. C. Busfield, and A. G. Thomas, J. Rubb. Res. 2, 88 (1999).

${ }^{22}$ O. H. Yeoh, Rubber Chem. Technol. 63, 792 (1990).

${ }^{23}$ M. Imbimbo and A. De Luca, Comp. Struct. 68, 31 (1998).

${ }^{24}$ P. Zoller and D. J. Walsh, "Standard Pressure-Volume-Temperature Data for Polymers," Lancaster, PA, Technomic, 1995.

${ }^{25}$ P. G. Tait, "Physics and Chemistry of the Voyage of H. M. S. Challenger," HMSO, London, Vol. 2, Part 4 (1888).

${ }^{26}$ L. A. Wood and G. M. Martin, J. Res. Nat. Bur. Stand. 68A, 259 (1964).

${ }^{27}$ W. K. Moonan and N. W. Tschoegl, Macromolecules 16, 55 (1983).

${ }^{28}$ W. L. Hergenrother, J. Appl. Polym. Sci. 32, 3039 (1986); 32, 3683 (1986).

${ }^{29}$ C. M. Roland, Rubber Chem. Technol. 62, 863 (1989).

${ }^{30}$ A. N. Gent. R. L. Henry, and M. L. Roxbury, J. Appl. Mech. 41, 855 (1974).

${ }^{31}$ R. S. Rivlin and D. W. Saunders, Phil. Trans. R. Soc. London Ser A, 243, 251 (1951).

${ }^{32}$ J. E. Mark, Polym. Eng. Sci. 19, 254 (1979).

${ }^{33}$ I.S. Choi and C.M. Roland, RubBer Chem. TeChNOL. 70, 202 (1997). 